\title{
Correntropy-based Analysis of Respiratory Patterns in Patients with Chronic Heart Failure
}

\author{
Ainara Garde, Student Member, IEEE, Leif Sörnmo, Senior Member, IEEE, Raimon Jané, Member, IEEE, \\ and Beatriz F. Giraldo, Member, IEEE
}

\begin{abstract}
A correntropy-based technique is proposed for the analysis and characterization of respiratory flow signals in chronic heart failure (CHF) patients with both periodic and nonperiodic breathing (PB and $\mathrm{nPB}$ ), and healthy subjects. Correntropy is a novel similarity measure which provides information on temporal structure and statistical distribution simultaneously. Its properties lend itself to the definition of the correntropy spectral density (CSD). An interesting result from CSD-based spectral analysis is that both the respiratory frequency and modulation frequency can be detected at their original positions in the spectrum without prior demodulation of the flow signal. The respiratory pattern is characterized by a number of spectral parameters extracted from the respiratory and modulation frequency bands. The results show that the power of the modulation frequency band offers excellent performance when classifying $\mathrm{CHF}$ patients versus healthy subjects, with an accuracy of $95.3 \%$, and $\mathrm{nPB}$ patients versus healthy subjects with $90.7 \%$. The ratio between the power in the modulation and respiration frequency bands provides the best results classifying $C H F$ patients into $\mathrm{PB}$ and $\mathrm{nPB}$, with an accuracy of $88.9 \%$.
\end{abstract}

\section{INTRODUCTION}

Patients with Cronical Heart Failure (CHF) often develop breathing anomalies, which include various forms of oscillatory breathing patterns characterized by rises and falls in ventilation. Periodic breathing (PB) patterns can be classified into ventilation with apnea, known as Cheyne-Stokes respiration (CSR), or ventilation without apnea [1], [2]. Some studies report a PB prevalence as high as $70 \%$ in these patients. Periodic breathing and CSR both have been related to increased mortality in CHF patients [3], [4] . Clinical studies have presented different physiological parameters for the detection of the above-mentioned respiratory patterns [5], [6]. The patterns are also influenced by wakefulness or sleep, posture, and physiological and mental activity [7].

Not only is the respiratory frequency essential when studying periodic and nonperiodic breathing patterns, but also the respiratory modulation frequency. Our first studies characterized the relevant frequency band determined by the

Manuscript received April 22, 2009. This work was supported in part by Ministerio de Ciencia e Innovación under grants TEC2007-68076-C02-01 and TEC2007-63637 from the Spanish Government.

A. Garde, B.F. Giraldo and R. Jané are with Dept. of ESAII, Universitat Politècnica de Catalunya (UPC), Institut de Bioenginyeria de Catalunya (IBEC) and CIBER de Bioingeniería, Biomateriales y Nanomedicina (CIBER-BBN). c/. Pau Gargallo, 5, 08028, Barcelona, Spain. (fax: +34 93401 7045) (e-mail: ainara.garde@upc.edu, beatriz.giraldo@upc.edu, raimon.jane@upc.edu).

L. Sörnmo is with the Department of Electrical and Information Technology and Center of Integrative Electrocardiology, Lund University, Lund SE-221 00, Sweden (e-mail: leif.sornmo@es.lth.se). peak extracted from the power spectral density (PSD) of the envelope of the respiratory flow signal [8], [9].

In this paper we propose a generalized autocorrelation function, known as correntropy, for the study of respiratory flow signal patterns in patients with CHF and healthy subjects. Correntropy is a similarity measure which contains information on both statistical distribution and temporal structure of the underlying dataset. The capability of preserving nonlinear characteristics makes the correntropy a suitable measure for determining nonlinear dynamics. Moreover, the use of kernel methods provides computational efficiency since it is possible to calculate the correntropy directly from the data [10]. The respiratory pattern is therefore characterized by the parameters extracted from the Correntropy Spectral Density (CSD).

This paper is organized as follows. Section II briefly presents the correntropy function, related parametric and nonparametric forms of the CSD, and the different parameters which are used to characterize the CSD. Section III describes the datasets used to evaluate method's performance, including both simulated and respiratory signals. The results are presented in Section IV.

\section{METHODS}

\section{A. Generalized Correlation Function-Correntropy}

Assuming stationarity, the correntropy function is defined as [10], [11]

$$
V(m)=E[\kappa(x(n)-x(n-m)],
$$

and can be estimated using

$$
\hat{V}(m)=\frac{1}{N-m+1} \sum_{n=m}^{N} \kappa(x(n)-x(n-m)),
$$

for each $n$ and $m \in N$, where $N$ is the number of samples, $E[\cdot]$ denotes the expectation operator, and $\kappa(\cdot)$ is a symmetric positive definite kernel function.

In the literature, sigmoidal, Gaussian, polynomial, and spline kernels are among the mostly used symmetric positive definite kernel functions in the area of machine learning, function approximation, density estimation, support vector machine, and others. The Gaussian kernel function is given by:

$$
\kappa(x(n)-x(n-m))=\frac{1}{\sqrt{2 \pi \sigma}} \exp \left\{-\frac{(x(n)-x(n-m))^{2}}{2 \sigma^{2}}\right\},
$$

where $\sigma$ is referred to as the kernel size parameter ("standard deviation"). We will apply the Gaussian kernel throughout the present paper. 
By means of the Taylor series expansion of the Gaussian kernel, we can rewrite the correntropy function as:

$$
V(m)=\frac{1}{\sqrt{2 \pi \sigma}} \sum_{k=0}^{\infty} \frac{(-1)^{k}}{\left(2 \sigma^{2}\right)^{k} k !} E\left[\left(x(n)-x(n-m)^{2 k}\right]\right.
$$

which contains all even-order moments of the random variable $(x(n)-x(n-m))$. Different kernel functions would yield different expansions, but all kernel functions mentioned above are nonlinear and therefore include higher order statistical information about the input random process.

Therefore, the correntropy function partially characterizes higher order statistics of random processes with a compact bivariate kernel function. The emphasis given to higherorder moments is controlled by the parameter $\sigma$, which in this study is determined by Silverman's rule [10] of density estimation:

$$
\sigma=0.9 A N^{-1 / 5}
$$

In this rule, $A$ is the smaller value between the standard deviation of the data samples and data interquartile range scaled by 1.34 , and $N$ is the number of samples. In the issue at hand, this technique provides a reasonable initial value since the signal is scalar.

As the respiratory frequency can range up to $0.5 \mathrm{~Hz}$, the respiratory flow signal, recorded at $250 \mathrm{~Hz}$ sampling rate, is downsampled to $2 \mathrm{~Hz}$. A wide variety of artifacts have been observed in flow signals, thus necessitating preprocessing to condition the signal. However, a powerful advantage of the correntropy function is its robustness against impulsive noise. This advantage is due to the fact that when an outlier is present, the inner product in the feature space computed via the Gaussian kernel tends to be zero (i.e., $\kappa(x(i)-x(i-k)) \approx$ 0 when either $x(i)$ or $x(i-k)$ is an outlier).

\section{B. Correntropy Spectral Density}

Similar to the conventional correlation function, it can be shown that correntropy is a positive definite function and therefore lends itself for many signal processing applications [12]. Since we are interested in exploring the spectral properties of the respiratory signals, such information is conveyed by the non parametric correntropy spectral density (CSD), defined by:

$$
P_{v}\left(e^{j \omega}\right)=\sum_{m=-\infty}^{\infty} V(m) e^{-j \omega m}
$$

A better spectral resolution can be obtained with parametric modeling. In particular, the autoregressive (AR) model is suitable for signals whose PSD contains pronounced spectral peaks. The extension of the correntropy to parametric CSD based on AR modeling is straightforward because it preserves the correlation properties.

\section{Parameter extraction}

The respiratory pattern is characterized by the CSD such that two frequency bands are defined, being centered around the respiratory frequency (" $\Delta f_{r}$ : the respiratory frequency band") and the modulation frequency (" $\Delta f_{m}$ : the modulation frequency band"). Taking into account that the modulation frequency of periodic breathing patterns ranges from 0.005 to $0.05 \mathrm{~Hz}$, the modulating frequency peak $\left(f p_{m}\right)$ is tracked around this interval with $\Delta f_{m}=0.02 \mathrm{~Hz}$. In addition, as the respiratory frequency ranges from 0.2 to $0.4 \mathrm{~Hz}$ its peak $\left(f p_{r}\right)$ is tracked around this interval with $\Delta f_{r}=0.2 \mathrm{~Hz}$. In order to characterize the two frequency bands, a number of parameters are evaluated for each band (see Table I).

TABLE I

PARAMETER DESCRIPTION

\begin{tabular}{cl} 
Parameter & Description \\
\hline$P_{m}$ & Power of the modulation frequency band \\
$S_{m}$ & Slope of the modulation frequency band \\
$P_{r}$ & Power of the respiratory frequency band \\
$S_{r}$ & Slope of the respiratory frequency band \\
$R$ & Relation between the $P_{m} / P_{r}$ \\
\hline
\end{tabular}

\section{DATASETS}

\section{A. Simulated Data}

We have observed that the behavior of the respiratory flow signal in PB patients resembles a signal subjected to amplitude modulation (AM). For these patients as well as for healthy subjects, the respiratory frequency usually ranges from 0.2 to $0.4 \mathrm{~Hz}$, whereas the modulation frequency ranges from 0.01 to $0.04 \mathrm{~Hz}$ [7]. In order to illustrate the method's performance, an AM signal is simulated whose carrier frequency is $f_{c}=0.3 \mathrm{~Hz}$ and modulation frequency is $f_{m}=0.02 \mathrm{~Hz}$, see Fig. 1 .

\section{B. Respiratory Data}

Respiratory flow signals were recorded from 35 healthy volunteers (12 males, 23 females, aged $26.6 \pm 7$ years) and 26 patients with CHF (19 males, 7 females, aged $65.0 \pm 9$ years) at the Santa Creu i Sant Pau Hospital in Barcelona, Spain. All subjects were studied according to a protocol previously approved by the local ethics committee. The respiratory flow signal was acquired using a pneumotachograph, consisting of a Datex-Ohmeda monitor with a Validyne Model MP451-871 Variable-Reluctance Transducer. The signals were recorded at $250 \mathrm{~Hz}$ sampling rate.

According to clinical criteria, $\mathrm{CHF}$ patients were classified into two groups: 8 patients with periodic breathing pattern and 18 patients with nonperiodic breathing pattern.

\section{RESUlTS}

\section{A. Illustration of the Method}

Fig. 2 illustrates the performance of the above-mentioned methods for power spectral estimation when the AM signal is analyzed. From this figure, it is evident that the spectra based on correntropy and correlation have very different characteristics, the main difference being related to the location of $f_{m}$. For the correntropy-based spectra, $f_{m}$ is found in the baseband and $f_{c}$ is accompanied with a number of harmonics. For the correlation-based spectra, the peaks are located at $f_{c}, f_{m}-f_{c}$ and $f_{m}+f_{c}$, respectively, as suggested 

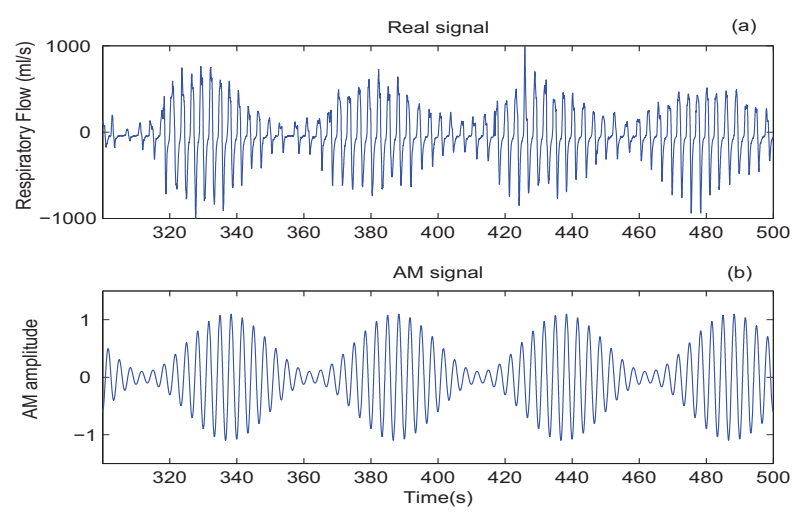

Fig. 1. The respiratory flow signal of (a) a PB patient, and (b) the simulated AM signal with $0.3 \mathrm{~Hz}$ carrier frequency and $0.02 \mathrm{~Hz}$ modulation frequency.
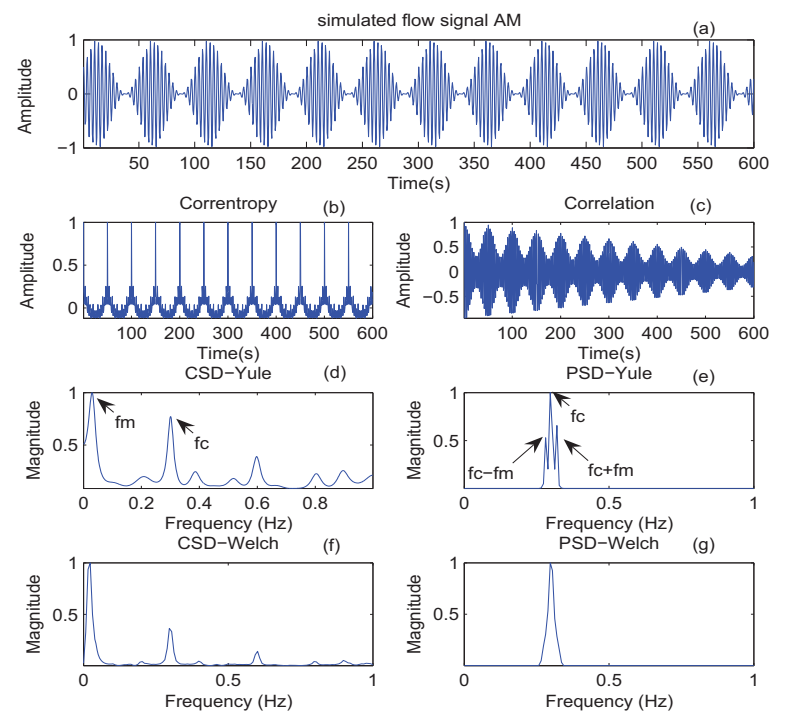

Fig. 2. (a) The simulated AM signal with $0.3 \mathrm{~Hz}$ carrier frequency and 0.02 $\mathrm{Hz}$ as modulation frequency, (b) its correntropy function and (c) correlation function. The correntropy spectral density obtained with (d) Yule-Walker's method and (f) Welch's method. The power spectral density obtained with (e) Yule-Walker's method and (g) Welch's method.

by classical AM analysis. Note that through Welch's method is unable to resolve the two peaks at $f_{m}-f_{c}$ and $f_{m}+f_{c}$, see Fig. 2(g); no harmonic pattern is present in the correlationbased spectra. Thus, correntropy-based spectra have the advantage of exhibiting peaks at the positions of $f_{m}$ and $f_{c}$ and can therefore be easily detected by some peak-searching algorithm. On the other hand, the sideband peaks of the correlation-based spectra are difficult to detect as they can smear with the respiratory peak at $f_{c}$.

Spectral analysis is also performed on real respiratory flow signals for the purpose of illustrating differences between the respiratory pattern showed by CHF patients with periodic breathing (Fig. 3) and nonperiodic breathing (Fig. 4), and healthy subjects (Fig. 5). In all types of spectra, the respiratory peak is clearly visible. The correntropy-based spectrum of the healthy subject (Fig. 5(d)) lacks a peak corresponding to the modulation frequency, unlike the spec-
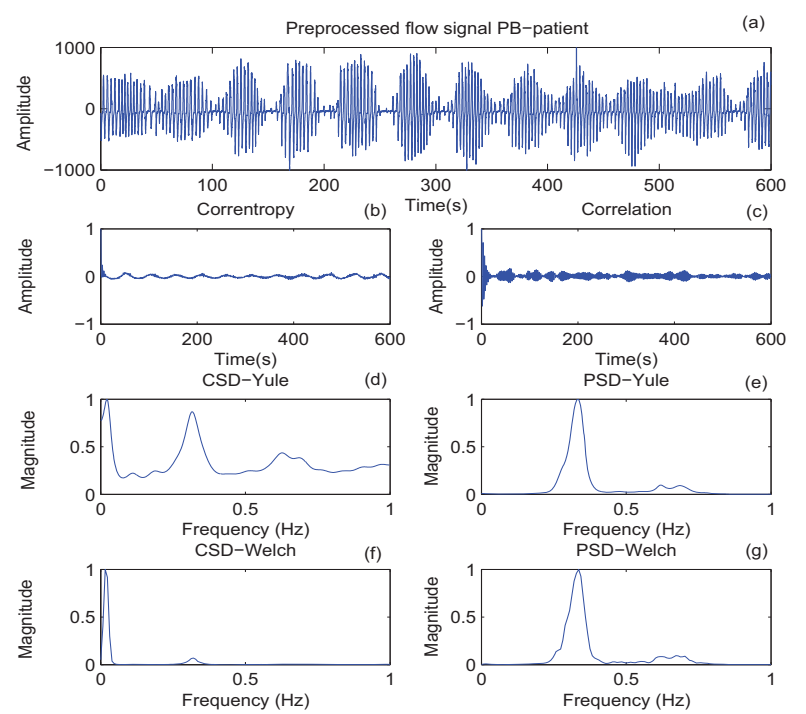

Fig. 3. (a) The respiratory flow signal of a CHF patient with periodic breathing, (b) its correntropy function and (c) correlation function. The correntropy spectral density obtained with (d) Yule-Walker's method and (f) Welch's method. The power spectral density obtained with (e) YuleWalker's method and (g) Welch's method.

tra showed by CHF patients. Periodic breathing patient presents a more powerful modulation peak than the non periodic one (Figs. 3(d) and 4(d)). Thus, for patients with periodic breathing, a modulation peak is easily detected in the correntropy-based spectra but not in the correlationbased spectra, Figs. 3(d) and 3(e). In fact, the latter type of spectrum does not lend itself to sideband peak detection.

\section{B. Performance Evaluation}

The characterization of respiratory flow patterns is evaluated in terms of the following three classification problems: CHF patients versus healthy subjects, nonperiodic breathing patients versus healthy subjects, and CHF patients with either periodic or nonperiodic breathing. The parameter selection process, being based on the leave-one-out crossvalidation, is implemented such that the most relevant parameter subset is selected. Since the dataset is small, a single parameter classification is consider instead of a multiparameter one. Table II shows the classification results achieved considering only the most discriminative parameter.

TABLE II

SENSITIVITY (SN), SPECIFICITY (SP), AND ACCURACY, OBTAINED WITH THE BEST PARAMETER FOR EACH CLASSIFICATION WITH LEAVE-ONE-OUT CROSSVALIDATION

\begin{tabular}{lcccc} 
Classifications & Parameter & Sn & Sp & Accuracy \\
\hline CHF vs. Healthy & $P_{m}$ & $96.3 \%$ & $94.3 \%$ & $95.3 \%$ \\
nPB-CHF vs. Healthy & $P_{m}$ & $94.7 \%$ & $88.6 \%$ & $90.7 \%$ \\
PB vs. nPB (CHF) & $R$ & $75.0 \%$ & $94.7 \%$ & $88.9 \%$
\end{tabular}



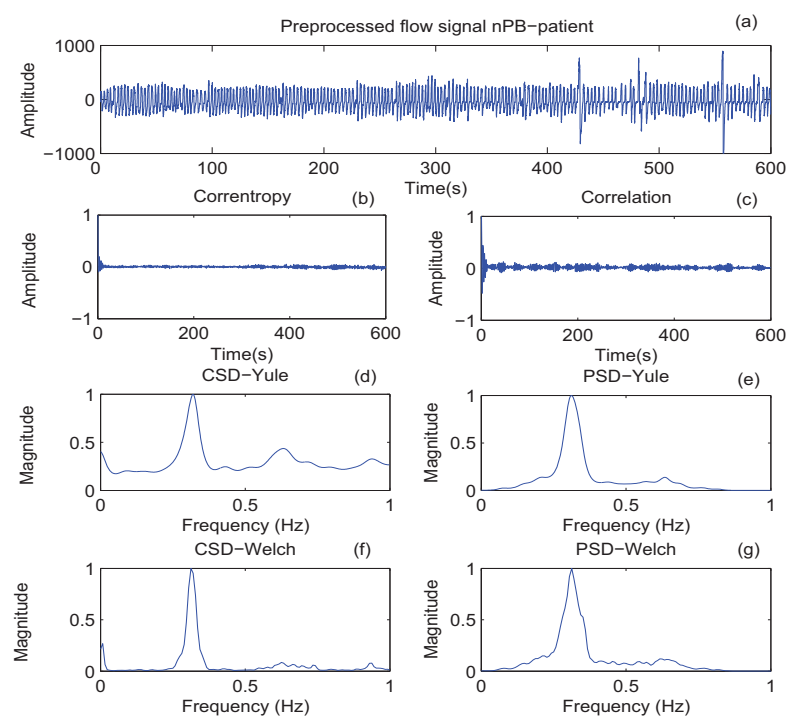

Fig. 4. (a) The respiratory flow signal of a CHF patient with nonperiodic breathing pattern, (b) its correntropy function and (c) correlation function. The correntropy spectral density obtained with (d) Yule-Walker's method and (f) Welch's method. The power spectral density obtained with (e) YuleWalker's method and (g) Welch's method.
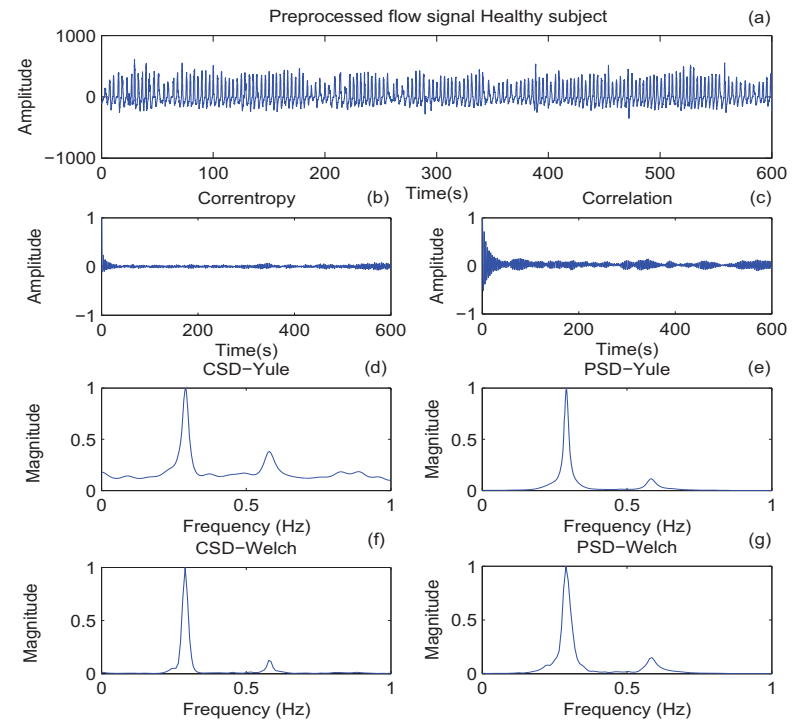

Fig. 5. (a) The respiratory flow signal of a healthy subject, (b) its correntropy function and (c) correlation function. The correntropy spectral density obtained with (d) Yule-Walker's method and (f) Welch's method. The power spectral density obtained with (e) Yule-Walker's method and (g) Welch's method.

\section{CONCLUSIONS}

In patients with periodic breathing, the correntropy-based spectrum exhibits peaks corresponding to respiration and modulation, being positioned at their true positions. As a result, spectral characterization of respiratory flow patterns is much facilitated. According to the parameters extracted from the frequency bands centered around the respiratory and modulation frequency peaks, power-related parameters provide excellent respiratory pattern characterization. The best result classifying CHF patients versus healthy subjects achieved an accuracy as high as $95.3 \%$, and classifying nPB patients versus healthy subjects an accuracy of $90.7 \%$, even if the power of the modulation frequency band is only considered. The ratio between the power in the modulation frequency band and the respiration frequency band provided good classification between $\mathrm{PB}$ and $\mathrm{nPB}$ into CHF patients, with an accuracy of $88.9 \%$. The study of patterns using correntropy should contribute with relevant information to enhance the prognosis of CHF patients, and may be used as a risk indicator. The results suggest that correntropy is a promising tool for classifying different respiratory patterns of CHF patients and healthy subjects. Further evaluation of the method's performance should be done on a much larger dataset.

\section{ACKNOWLEDGMENTS}

The authors would like to thank to Drs. S. Benito and A. Bayés-Genis and their collaborators of Santa Creu i Sant Pau Hospital, Barcelona, Spain, for their collaboration in the signal database acquisition.

\section{REFERENCES}

[1] G. Lorenzi-Filho, P. R. Genta, A. C. Figueiredo, and D. Inoue, "Cheyne-stokes respiration in patients with congestive heart failure: Causes and consequences," Clinics (Sao Paulo, Brazil), vol. 60, pp. 333-344, 2005.

[2] D. P. Francis, K. Wilson, L. C. Davies, A. J. Coats, and M. Piepoli, "Quantitative general theory for periodic breathing in chronic heart failure and its clinical implications," Circulation, vol. 102, pp. 2214 2221, 2000.

[3] G. D. Pinna, R. Maestri, A. Mortara, M. T. L. Rovere, F. Fanfulla, and P. Sleight, "Periodic breathing in heart failure patients: testing the hypothesis of instability of the chemoreflex loop," J. Appl. Physiol., vol. 89, pp. 2147-2157, 2000.

[4] P. J. Hanly and N. S. Zuberi-Khokhar, "Increased mortality associated with cheyne-stokes respiration in patients with congestive heart failure," American journal of respiratory and critical care medicine, vol. 153, no. 1, pp. 272-276, 1996.

[5] J. P. Ribeiro, "Periodic breathing in heart failure: bridging the gap between the sleep laboratory and the exercise laboratory," Circulation, vol. 113, pp. 9-10, 2006.

[6] P. Ponikowski, S. D. Anker, T. P. Chua, D. Francis, W. Banasiak, P. A. Poole-Wilson, A. J. Coats, and M. Piepoli, "Oscillatory breathing patterns during wakefulness in patients with chronic heart failure: clinical implications and role of augmented peripheral chemosensitivity,' Circulation, vol. 100, pp. 2418-2424, 1999.

[7] G. D. Pinna, R. Maestri, A. Mortara, and M. T. La Rovere, "Cardiorespiratory interactions during periodic breathing in awake chronic heart failure patients," Am. J. Physiol. (Heart Circ. Physiol.), vol. 278, pp. H932-941, 2000.

[8] A. Garde, B. Giraldo, R. Jané, I. Diaz, S. Herrera, S. Benito, M. Domingo, and A. Bayes-Genis, "Analysis of respiratory flow signals in chronic heart failure patients with periodic breathing," in Proc. IEEE Conf. Eng. Med. Biol., 2007, pp. 307-310.

[9] A. Garde, B. F. Giraldo, R. Jané, I. Diaz, S. Herrera, S. Benito, M. Domingo, and A. Bayes-Genis, "Characterization of periodic and non-periodic breathing pattern in chronic heart failure patients," in Proc. IEEE Conf. Eng. Med. Biol., 2008, pp. 3227-3230.

[10] I. Santamaria, P. P. Pokharel, and J. C. Principe, "Generalized correlation function: definition, properties, and application to blind equalization," Signal Processing, IEEE Transactions on, vol. 54, no. 6, pp. 2187-2197, 2006.

[11] J. W. Xu and J. C. Principe, "A pitch detector based on a generalized correlation function," Audio, Speech, and Language Processing, IEEE Transactions on, vol. 16, pp. 1420-1432, 2008.

[12] W. Liu, P. P. Pokharel, and J. C. Principe, "Correntropy: Properties and applications in non-gaussian signal processing," Signal Processing, IEEE Transactions on, vol. 55, no. 11, pp. 5286-5298, 2007. 